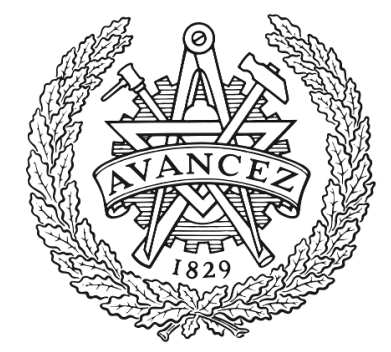

\title{
CHALMERS
}

UNIVERSITY OF TECHNOLOGY

\section{Hot-Carrier Generation in Plasmonic Nanoparticles: The Importance of Atomic Structure}

Downloaded from: https://research.chalmers.se, 2023-04-26 14:28 UTC

Citation for the original published paper (version of record):

Rossi, T., Erhart, P., Kuisma, M. (2020). Hot-Carrier Generation in Plasmonic Nanoparticles: The Importance of Atomic Structure. ACS Nano, 14(8): 9963-9971.

http://dx.doi.org/10.1021/acsnano.0c03004

N.B. When citing this work, cite the original published paper. 


\title{
Hot-Carrier Generation in Plasmonic Nanoparticles: The Importance of Atomic Structure
}

\author{
Tuomas P. Rossi, * Paul Erhart, ${ }^{*}$ and Mikael Kuisma*
}

Cite This: ACS Nano 2020, 14, 9963-9971

Read Online

ABSTRACT: Metal nanoparticles are attractive for plasmon-enhanced generation of hot carriers, which may be harnessed in photochemical reactions. In this work, we analyze the coherent femtosecond dynamics of photon absorption, plasmon formation, and subsequent hot-carrier generation through plasmon dephasing using first-principles simulations. We predict the energetic and spatial hot-carrier distributions in small metal nanoparticles and show that the distribution of hot electrons is very sensitive to the local structure. Our results show that surface sites exhibit enhanced hot-electron generation in comparison to the bulk of the

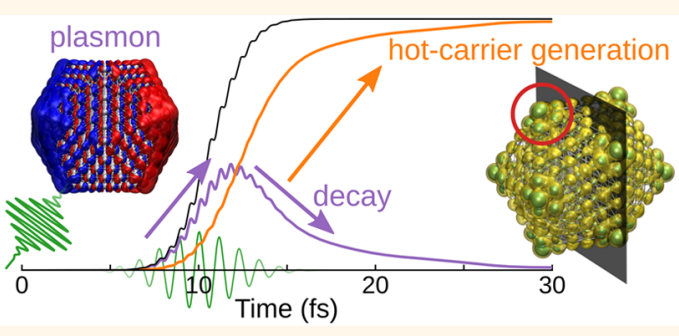
nanoparticle. Although the details of the distribution depend on particle size and shape, as a general trend, lower-coordinated surface sites such as corners, edges, and $\{100\}$ facets exhibit a higher proportion of hot electrons than higher-coordinated surface sites such as $\{111\}$ facets or the core sites. The present results thereby demonstrate how hot carriers could be tailored by careful design of atomic-scale structures in nanoscale systems.

KEYWORDS: localized surface plasmon, plasmon decay, plasmon dephasing, time-dependent density functional theory, hot electrons, hot carriers, atomic scale

$\mathrm{P}$ lasmon-enhanced technologies enabled by metal nanoparticles (NPs) provide promising avenues for harvesting and converting sunlight to chemical energy ${ }^{1}$ and driving photochemical reactions. ${ }^{2}$ The underlying processes rely on the decay of plasmonic excitations and the subsequent generation of high-energy non-equilibrium electrons and holes. ${ }^{3}$ These electrons and holes are often collectively referred to as hot carriers ( $\mathrm{HCs})$, but their distributions can vary substantially with time after excitation. ${ }^{4,5} \mathrm{HCs}$ generated by plasmon decay can, in principle, be transferred to a chemically attached acceptor such as a semiconductor or a molecule, a process that is potentially useful for technologies such as photovoltaics, ${ }^{6}$ photodetection, ${ }^{7,8}$ photon up-conversion, ${ }^{9}$ and photocatalysis ${ }^{2,10-13}$ and possibly relevant for NP growth processes. ${ }^{14}$

It can be challenging to develop comprehensive understanding of plasmon-generated hot carriers via purely experimental approaches both due to time constraints and the difficulty associated with disentangling different contributions. ${ }^{15-17}$ In this context, complementary theoretical and computational approaches can provide highly valuable insight as they enable scrutinizing the relevant microscopic processes. The present theoretical understanding of plasmonic $\mathrm{HC}$ generation is mostly based on flat metal surfaces ${ }^{18,19}$ or jellium NPs neglecting the underlying atomic structure. ${ }^{4,5,20-26}$ Whereas atomic-scale effects in nanoplasmonics, in general, have been increasingly addressed in recent years, ${ }^{27-32}$ atomic-scale modeling of plasmonic HC generation is only emerging. ${ }^{33-35}$ In particular, detailed atomic-scale distributions of plasmongenerated HCs, to our knowledge, have not yet been scrutinized. In the context of photocatalysis detailed understanding of plasmonic HC generation at the atomic scale is, however, of paramount importance as chemical reactions take place at this size scale.

In this work, we analyze the effect of local atomic-scale structure on plasmonic $\mathrm{HC}$ generation and demonstrate that the distribution of HCs after plasmon decay is sensitive to the atomic-scale details. Using a series of NPs, we analyze quantitatively the spatial distribution of plasmon-generated $\mathrm{HCs}$ at the atomic scale with respect to surface orientation as well as for edge and corner sites. As the trends are consistent across NPs of different size and shape, the trends obtained here are expected to be transferable to more general nanoscale

Received: April 9, 2020

Accepted: July 20, 2020

Published: July 20, 2020

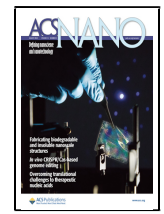


(a)

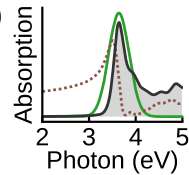

(b)

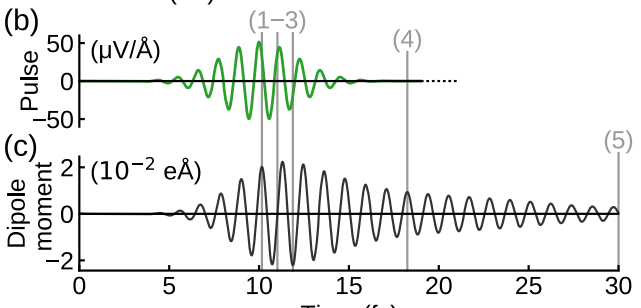

(d)

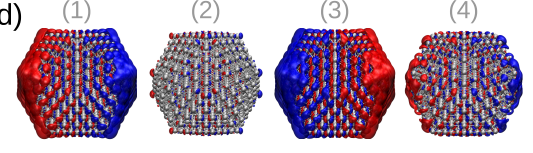

(e)

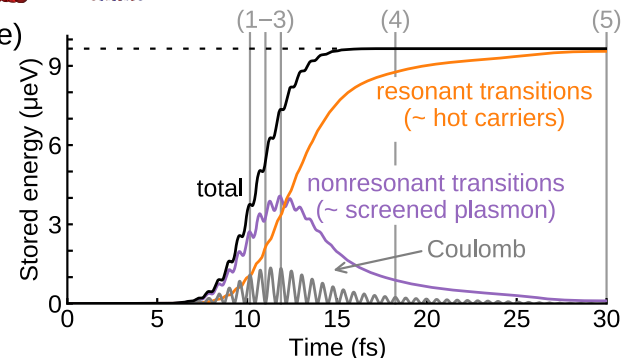

Figure 1. Real-time dynamics of a localized surface plasmon in a silver nanoparticle. (a) Photoabsorption spectrum of the Ag ${ }_{561} \mathrm{NP}$ (shaded) and the intensity profile of an impacting electric field pulse (green). Photoabsorption is determined by the imaginary part of the polarizability, $\alpha$, and the corresponding real part, $\operatorname{Re}[\alpha]$, is shown as a dotted line. (b) Electric field pulse impacting the plasmon resonance of the NP. (c) Time-dependent dipole moment response of the NP. (d) Electron density oscillations in the NP at selected time instances (red and blue isosurfaces denote density increase and decrease, respectively). (e) Time evolution of the energy stored in the excited electronic system. The total energy (black) is divided into the energy of nonresonant electron-hole transition contributions constituting screened plasmon excitation (purple) and that of resonant transition contributions constituting mainly hot carriers (orange). A part of the plasmon energy is in the form of Coulomb energy (gray).

structures. For this study, we have developed a fully atomistic, parameter-free, and generally applicable description of plasmonic HC generation based on Kohn-Sham (KS) density functional theory (DFT) $)^{36,37}$ and time-dependent density functional theory (TDDFT). ${ }^{38}$

\section{RESULTS AND DISCUSSION}

Real-Time Dynamics of Localized Surface Plasmon. To introduce our approach for modeling plasmonic HC generation, we start with a comprehensive characterization of plasmon formation and subsequent dephasing. We consider an icosahedral $\mathrm{Ag}_{561}$ silver NP as an example system with a clear plasmon resonance in the photoabsorption spectrum (Figure 1a). ${ }^{39}$ The ground-state electronic structure of the NP is calculated with DFT using the Gritsenko-van Leeuwen-van Lenthe-Baerends-solid-correlation (GLLB-sc) exchange-correlation (XC) potential ${ }^{40,41}$ for an improved d-band description. ${ }^{28,42}$ The time-dependent response is then calculated with TDDFT using either the random-phase approximation (RPA) or the adiabatic GLLB-sc ${ }^{28}$ (see Methods for details).

To excite the localized surface plasmon resonance (LSPR) in the NP, we use a monochromatic ultrafast Gaussian light pulse

$$
\mathcal{E}(t)=\mathcal{E}_{0} \cos \left(\omega_{0}\left(t-t_{0}\right)\right) \exp \left(-\left(t-t_{0}\right)^{2} / \tau_{0}^{2}\right)
$$

that induces real-time dynamics of electrons in the system. The pulse frequency $\omega_{0}=3.6 \mathrm{eV}$ is tuned to the plasmon resonance, the pulse duration is determined by $\tau_{0}=3 \mathrm{fs}$, and the pulse is centered at $t_{0}=10 \mathrm{fs}$ (Figure $1 \mathrm{~b}$ ). The pulse strength is weak, $\mathcal{E}_{0}=51 \mu \mathrm{V} / \AA$, putting the response in the linear-response regime. In the frequency space, the pulse is wide enough to cover the whole plasmon resonance (Figure 1a).

The interaction between electrons and light is described in the dipole approximation, within which the light pulse creates a time-dependent external potential, $v_{\text {pulse }}(t)=z \mathcal{E}(t)$, that causes the time evolution of the KS states $\left|\psi_{n}(t)\right\rangle$ and excitation of the LSPR. The light pulse induces a strong dipole-moment response [Figure 1c $(1-3)]$. The corresponding electron density oscillations [Figure $1 \mathrm{~d}(1-3)]$ are composed of a surface-tosurface component associated with delocalized valence electrons near the Fermi energy and atom-localized contributions that correspond to screening due to virtual excitations from the dband. ${ }^{39}$ As time proceeds to $t \approx 17 \mathrm{fs}$, the excited electrons start to lose their collective plasmonic motion via a dephasing process commonly referred to as Landau damping. ${ }^{43}$ As the plasmon dephases, the dipole moment decays [Figure 1c $(4,5)$ ] corresponding to vanishing surface-to-surface density oscillation [Figure $1 \mathrm{~d}(4,5)] .{ }^{33}$ Whereas the density oscillations and dipole moment response gives an illustrative picture of the plasmon formation and decay, they seem not to provide a tractable way for scrutinizing $\mathrm{HC}$ contributions to the response. To this end, we consider the time-dependent energy contributions of different KS transitions.

Time-Dependent Energy Contributions. As the pulse is tuned to the LSPR, the electronic system absorbs energy from the incident light and remains in an excited state after the pulse has vanished. To analyze the distribution of this energy, we first consider the total time-dependent energy of the system given by

$$
E_{\text {tot }}(t)=E_{\text {tot }}^{(0)}+\Delta E(t)+E_{\text {pulse }}(t)
$$

where $E_{\text {tot }}^{(0)}$ is the ground-state energy, $\Delta E(t)$ is the timedependent energy stored in the excited state (Figure 1e, black line), and $E_{\text {pulse }}(t)=-\mu(t) \mathcal{E}(t)$ is the potential energy of the system under the external electric field.

The incident light pulse pumps energy into the system; that is, it does work on the system as $\Delta \dot{E}(t)=\delta \dot{\mu}(t) \mathcal{E}(t)$, where dots indicate time derivatives and $\delta \mu(t)=\mu(t)-\mu^{(0)}$ is the induced dipole moment. Thus, the total accumulated electronic energy can be written as

$$
\Delta E(t)=\int_{0}^{t} \delta \dot{\mu}(\tau) \mathcal{E}(\tau) \mathrm{d} \tau
$$

The electronic energy increases through absorption in a stepwise manner following the pulse intensity (Figure $1 \mathrm{e}, t \approx 5 \ldots 15 \mathrm{fs}$ ). After the pulse has ended ( $t \gtrsim 15 \mathrm{fs}$ ), the absorbed energy remains in the system and the total energy has attained a new constant value given by the photoabsorption cross section (Figure 1a and eq 11 in Methods).

Although the total energy remains constant, the electronic energy does not stay equally distributed among the electronhole transitions $i \rightarrow a$ excited by the light pulse. To quantify this 


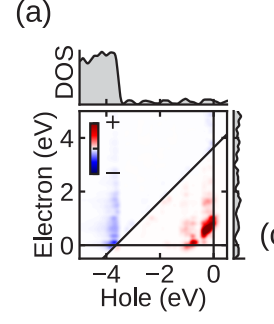

(b)

(1) $10.2 \mathrm{fs}$

(2) $11.0 \mathrm{fs}$

(3) $11.9 \mathrm{fs}$

(4) 18.2 fs

(5) $30.0 \mathrm{fs}$
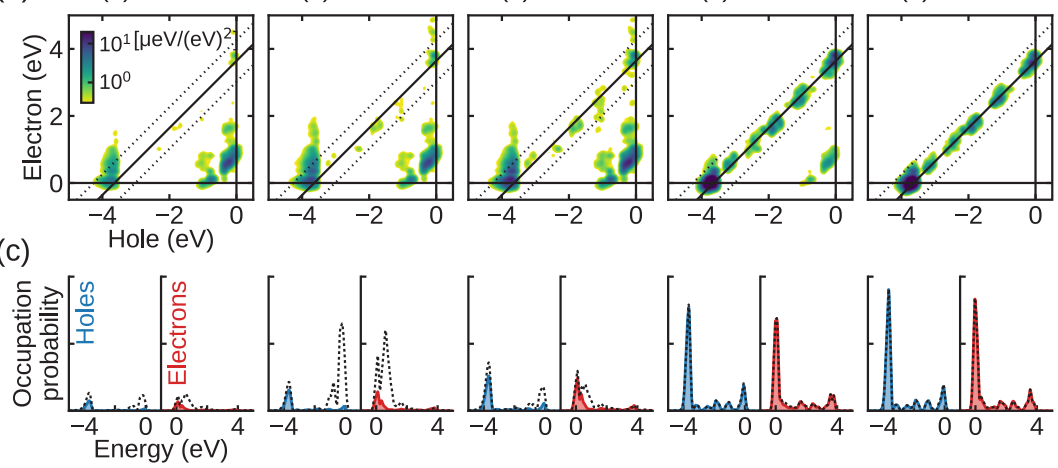

Figure 2. Electron-hole transition contributions to plasmon formation and decay. (a) Electron-hole contributions to the photoabsorption at the resonance energy visualized as a transition contribution map (TCM). Density of states is also shown along the energy axes. (b) Electronhole contributions to the time-dependent electronic energy visualized as TCM on a logarithmic color scale. The solid diagonal line corresponds to the transition energies matching with the pulse frequency $\omega_{0}$ and the dotted diagonal lines are drawn at $\omega_{0} \pm 2 \sigma$ to indicate the pulse width $\sigma=\sqrt{2} / \tau_{0}$, defining the boundaries for resonant and nonresonant transitions for Figure 1e. (c) Occupation probabilities of hole and electron states. Solid blue and red lines denote state occupations from resonant transitions, and dashed lines denote occupations from all transitions (resonant and nonresonant). The figure columns (1-5) show panels (b) and (c) for the time instances labeled in Figure 1 . The color scale and axis limits are the same in all of the columns.

effect, we consider the decomposition of the energy in electronhole transition contributions. This decomposition is based on the linear response of the KS density matrix, $\delta \rho_{i a}(t)$, expressed in the basis of the eigenstates $\left(\epsilon_{n}, \psi_{n}^{(0)}\right)$ of the ground-state Hamiltonian. The electron-hole decomposition of energy is (see Supplementary Note S1 for derivation)

$$
\Delta E(t)=\sum_{i a}^{f_{i}>f_{a}} \omega_{i a} P_{i a}(t)+E_{i a}^{\mathrm{C}}(t)
$$

where the sum is restricted by ground-state occupation numbers $f_{n}$ so that the indices $i$ and $a$ correspond to the created hole and electron states, respectively. Here, $\omega_{i a}=\epsilon_{a}-\epsilon_{i}$ is the electronhole transition energy (the KS eigenvalue difference), $P_{i a}(t)$ is the transition probability defined as

$$
P_{i a}(t)=\left|\frac{\delta \rho_{i a}(t)}{\sqrt{f_{i}-f_{a}}}\right|^{2}
$$

and $E_{i a}^{\mathrm{C}}(t)$ is the Coulomb energy as obtained from the HartreeXC kernel (defined in Supplementary Note S1).

Plasmon formation and dephasing can be scrutinized by considering the energy stored in the electronic system in terms of the electron-hole transition energy, $\omega_{i a}$, with respect to the pulse frequency $\omega_{0}$ (Supplementary Figure S1). The plasmon is formed by constructive coupling of low-energy transitions $\left[\omega_{i a}\right.$ $\lesssim 2 \mathrm{eV}$; see Figure $2 \mathrm{a}$ and time instances $(1-3)$ in Figure $2 \mathrm{~b}$. $^{39,43,44}$ Simultaneously, high-energy virtual d-electron transitions $\left(\omega_{i a} \gtrsim 4 \mathrm{eV}\right)$ screen the plasmonic density oscillation, decreasing the total induced field. The energy stored in the screening is similar to the energy stored in the polarization of a dielectric in general; that is, as the total field strength decreases, energy is instead stored in the polarization of the d-electron states as in $\omega_{i a} P_{i a}(t)$ in eq 4 . The nonresonant low- and highenergy transitions carry most of the energy during plasmon excitation (Figure 1e, purple line). As the plasmon dephases, the absorbed energy is redistributed to electron-hole transitions that are resonant with the pulse [Figure 1e, orange line; corresponding to the diagonal in the transition contribution maps in Figure $2 b$; see time instances $(4,5)]$. After dephasing, the energy remains almost exclusively stored in these transitions constituting the plasmon-generated HCs. The transitions comprising the plasmon are active in photoabsorption (Figure $2 \mathrm{a}$ ), and hot holes and electrons are generated through plasmon decay, instead of the HC transitions absorbing the light directly (shown in detail in Supplementary Figure S2).

At longer time scales, the electronic system would dissipate the absorbed energy to the environment via radiation, atomic motion, or other processes, but such decay pathways are not included within the description used here. Thus, in the present picture, there appears no significant dynamics in $\mathrm{Ag}_{561}$ at time scales beyond $t \gtrsim 30 \mathrm{fs}$ after the fast dephasing of the LSPR through Landau damping, which takes place due to the presence of multiple excitation eigenstates forming the broadened plasmon peak in the photoabsorption spectrum ${ }^{43}$ (Supplementary Figure S3). However, the dynamics can be very different in small clusters with a discrete excitation spectrum. In contrast to a single broad plasmon peak in $\mathrm{Ag}_{561}$, for example, in the $\mathrm{Ag}_{55}$ cluster, individual electron-hole transitions couple strongly to the plasmon, ${ }^{39}$ a process that is referred to as plasmon fragmentation. ${ }^{45,46}$ Correspondingly, the time-domain response exhibits Rabi oscillations and energy transfer back from the resonant transitions to the plasmon ${ }^{33,47}$ (Supplementary Figure S4).

As the coupling of transitions via Coulomb interaction is recognized as an essential characteristic of plasmonic excitations, ${ }^{39,43,44,48}$ it is instructive to consider the Coulomb energy, $E_{\mathrm{C}}(t)=\sum_{i a} E_{i a}^{\mathrm{C}}(t)$. This energy exhibits strong oscillations (Figure 1e, gray line) analogous to the dipole moment (Figure 1c) as only the electron density oscillation contributes to the Coulomb energy. At the maxima of the surface-to-surface density oscillation [time instances (1) and (3) in Figure 1d,e], the Coulomb contribution is a significant part of the plasmon energy, but at the minima between [e.g., time instance (2)], the Coulomb energy is vanishing as the electronic energy is stored in the electron current flowing through the particle (Supplementary Figure S5).

Temporal Evolution of Hot-Carrier Distributions. Having established a real-time picture of plasmon formation and decay, we are in the position to analyze the distributions of 

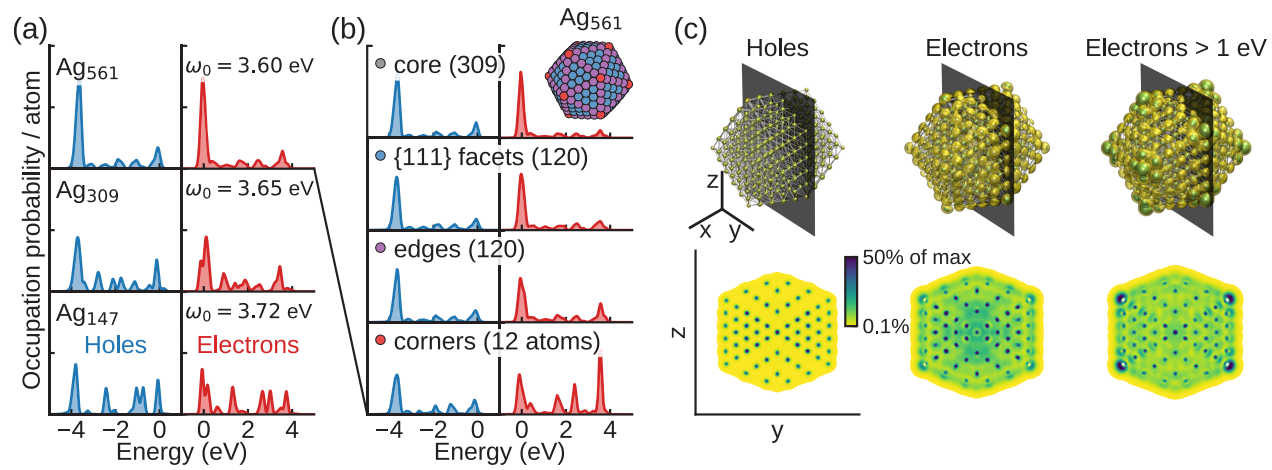

Figure 3. Hot-carrier distributions after plasmon decay. (a) Occupation probabilities of hole and electron states in icosahedral silver NPs of 147-561 atoms. (b) Occupation probabilities at different atomic sites of $\mathrm{Ag}_{561}$. All the panels use the same axis limits (normalized by the number of atoms). (c) Spatial density profiles of all induced holes and electrons and induced electrons with energy of more than $1 \mathrm{eV}$ in $\mathrm{Ag}_{561}$. Plots show isosurfaces corresponding to 10 and $20 \%$ of maximum value, and slices are taken through the center of the NP.

electrons and holes during the process. The probabilities for creating a hole in an initially occupied state $i$ or an electron in an initially unoccupied state $a$ are given directly by the transition probability of eq 5 as

$$
P_{i}^{\mathrm{h}}(t)=\sum_{a}^{f_{i}>f_{a}} P_{i a}(t) \text { and } P_{a}^{\mathrm{e}}(t)=\sum_{i}^{f_{i}>f_{a}} P_{i a}(t)
$$

respectively. $P_{i}^{\mathrm{h}}$ and $P_{a}^{\mathrm{e}}$ determine exactly the diagonal elements of the second-order response of the density matrix (Supplementary Note S1); in other words, they correspond to the increase of the occupation of the initially unoccupied state $a$ and the decrease of the occupation of the initially occupied state $i$.

The occupation probabilities given by eq 6 show strong oscillations during the time evolution [Figure $2 c(1-3)$, dashed lines]. These oscillations are explained by the oscillation of the Coulomb energy. As the Coulomb energy contribution is carried mainly by nonresonant transitions (Supplementary Figure S6), the occupation probabilities of the electron and hole states contributing to these nonresonant transitions oscillate analogously to the Coulomb energy. The oscillations are especially visible in the occupations of electron and hole states that form the plasmon, ${ }^{35}$ that is, the states near the Fermi energy, often referred to as Drude carriers. ${ }^{49}$ The oscillatory population and depopulation of these states indicate that they would not likely be individually separable while they are a part of the plasmon as the Coulomb interaction is an essential part of the excitation itself. $^{50}$

The contributions of the resonant transitions to the Coulomb energy are relatively small (Supplementary Figure S6), and the occupations of the corresponding electron and hole states grow steadily as the plasmon decays (Figure $2 c$, solid lines). At the end of the dynamic evolution studied here [Figure $2 c(5)$ ], electrons and holes are still coupled in the form of electron-hole transitions, and the distributions at $t=30 \mathrm{fs}$ can be considered as the initial nonthermal HC distributions. After their generation, these carriers would separate and interact via electron-electron and electron-phonon scattering processes ${ }^{3,5,19,51}$ that are not captured in the present description. The slight asymmetry between the hole and electron distributions is caused by a nonzero width of the pulse in the frequency space (Figure 1a).

Energetic and Atomic-Scale Spatial Distributions of Hot Carriers. We now analyze the distribution of plasmongenerated hot carriers and the impact of local atomic-scale structure. We start by considering the series of icosahedral silver
NPs $\mathrm{Ag}_{147}, \mathrm{Ag}_{309}$, and $\mathrm{Ag}_{561}$, the photoabsorption properties of which we have described in detail in previous work ${ }^{28,39}$ (see Supplementary Figure S7 for photoabsorption spectra and densities of states). The light pulse is tuned to the plasmon resonance of the NPs, and the initial HC distributions are analyzed after the plasmon has dephased at a time $t=30 \mathrm{fs}$. The $\mathrm{HC}$ distributions show a pronounced dependence on NP size (Figure $3 \mathrm{a}$ ) and local structure (Figure $3 \mathrm{~b}, \mathrm{c}$ ) as discussed in the following.

As particle size increases, the $\mathrm{HC}$ distributions are increasingly dominated by interband d-electron transitions ${ }^{26}$ (hole $\sim-4 \mathrm{eV} \rightarrow$ electron $\sim 0 \mathrm{eV}$ ) converging toward the distributions obtained for flat surfaces. ${ }^{18,19}$ In contrast to extended systems, geometry confinement effects are significant for plasmonic $\mathrm{HC}$ generation in nanoscale systems. ${ }^{52}$ Due to the broken crystal symmetry, additional "intraband" transitions are available for plasmonic HC generation in NPs, which results in the population of higher-energy electron and hole states (Figure 3a; electron states of $>0.5 \mathrm{eV}$, hole states of $>-3.5 \mathrm{eV}$ ). The relative contribution of these sp-states is most pronounced in the smallest $\mathrm{NPs}^{26}\left(\mathrm{Ag}_{147}, \mathrm{Ag}_{309}\right)$, but they are non-negligible also in $\mathrm{Ag}_{561}$. Similar size-dependent trends are also present in silver NPs of other shapes, whereas the detailed relative contributions of different transitions vary (Supplementary Figure S8).

The calculated probability distributions of plasmon-generated electrons and holes (see Methods) exhibit strong spatial variance (shown for the icosahedral $\mathrm{Ag}_{561} \mathrm{NP}$ in Figure $3 \mathrm{~b}, \mathrm{c}$ ): Holes are localized at atomic sites throughout the particle, which is expected as the majority of holes originates from the atomlocalized d-states. As a result, their energy distribution is very similar for core and surface sites. Hot electrons, on the other hand, are more delocalized and reside to larger extent in the surface region. The surface contribution is even more pronounced for higher-energy hot electrons ( $>1 \mathrm{eV}$ electrons in Figure 3c). The probability density of these hot electrons is strongly enhanced especially at low-coordinated edge and corner sites compared to sites in the core and on flat surfaces (Figure $3 \mathrm{~b}$ ). The energetic distributions of plasmon-generated holes and electrons are not necessarily symmetric when projected onto a particular site (Figure $3 \mathrm{~b}$ and Supplementary Figure S8). This asymmetry is especially pronounced for the corner site in $\mathrm{Ag}_{561}$, which reflects the fact that the hot-electron density at corner sites originates likely from throughout the particle due to the uniformity of the hole density. 

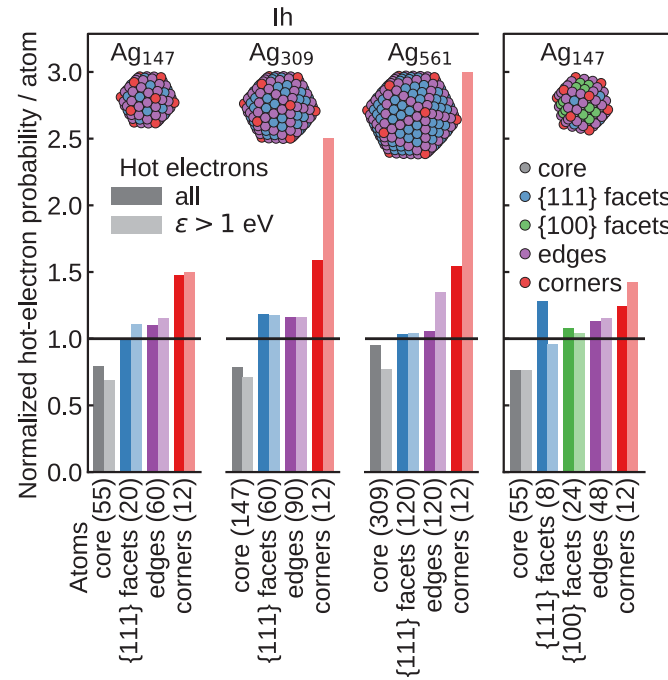

Cub-Oh
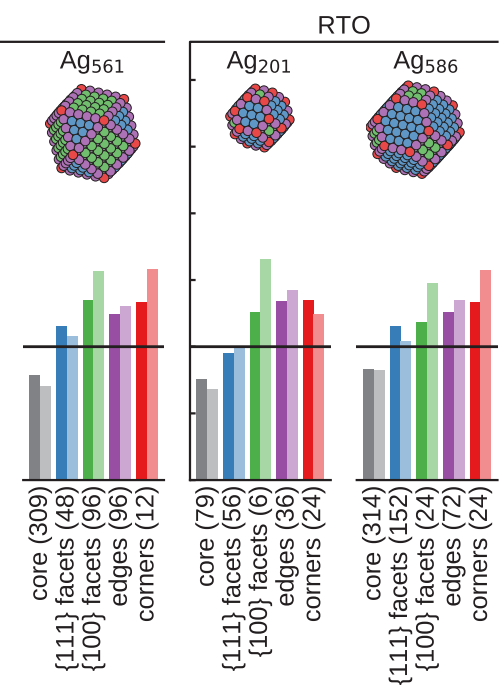

Figure 4. Atomic-scale distributions of hot electrons in silver nanoparticles. Spatial distribution of hot electrons generated on different atomic sites in icosahedral (Ih), cuboctahedral (Cub-Oh), and regularly truncated octahedral (RTO) NPs. Sites with lower coordination exhibit a higher proportion of hot electrons than core sites. A spatially uniform distribution corresponds to a normalized probability of unity. The insets show the atomic structures with the different atomic sites colored.

A more quantitative view is obtained by considering the total per-atom occupation probability of hot electrons at a particular atomic site in comparison to the total per-atom occupation probability throughout the system (Figure 4). These data suggest that in the icosahedral $\mathrm{Ag}_{561} \mathrm{NP}$, hot electrons with more than $1 \mathrm{eV}$ are almost three times more likely to be found in the vicinity of a corner site than a uniform spatial distribution would correspond to. We emphasize that these are per-atom considerations; that is, as the 12 corner atoms constitute only around $2 \%$ of the atoms in the particle, it is expected that about $6 \%$ of the electrons with more than $1 \mathrm{eV}$ would be generated in the vicinity of the corner atoms. Overall, hot electrons with more than $1 \mathrm{eV}$ constitute $30-60 \%$ of all hot electrons depending on system (Supplementary Figure S8). The absolute total number of HCs generated depends on the light energy that is absorbed, which is in turn determined by the photoabsorption cross section and light intensity.

Similarly to icosahedral shapes, hot electrons in the cuboctahedral and regularly truncated octahedral NPs are more likely to be generated at a surface site than at a core site (Figure 4). The preference for surface sites is even more pronounced for hot electrons with more than $1 \mathrm{eV}$. The corner sites of the cuboctahedral and regularly truncated octahedral NPs, however, do not show as enhanced distribution of hot electrons as those sites of the icosahedral $\mathrm{Ag}_{561}$ particle. This further underlines the sensitivity of $\mathrm{HC}$ generation to atomicscale details and the exact electronic structure of the NP and site(s) in question.

As a general trend, lower-coordinated sites seems to exhibit an enhancement of hot electrons compared to higher-coordinated sites. This trend is observed for corner and edge sites, but the data for cuboctahedral and regularly truncated octahedral NPs suggest also that more hot electrons are generated on the lowercoordinated $\{100\}$ surface than on the $\{111\}$ surface. In contrast to strong spatial variation of hot electrons, plasmon-generated holes do not show strong spatial dependence in the considered NPs (Supplementary Figure S9).

For practical utilization, HCs need to be transferred to the environment. The HC distributions obtained in the present work can be considered as the initial nonthermal $\mathrm{HC}$ distributions before any electron-electron scattering ${ }^{19,51}$ has taken place. Thus, these HC distributions could serve as an initial condition for subsequent dynamics ${ }^{3,5}$ that is not included in the present approach. In general, $\mathrm{HC}$ transfer to environment can occur indirectly (i.e., carriers are first produced in the metal and subsequently transferred to the acceptor) or directly (i.e., plasmon dephasing leads directly to the injection of HCs in empty acceptor, or occupied donor, states), ${ }^{2,53-62}$ However, both experiments ${ }^{63-65}$ and calculations ${ }^{51,52,65,66}$ indicate that HCs generated in the metal can quickly relax through electronelectron scattering, which renders the indirect pathway inefficient. The direct-transfer process, on the other hand, presents an opportunity to obtain more efficient plasmonic HC devices. $^{54,56,62}$ The prevalence of hot electrons on lowercoordinated surface sites described in the present work seems favorable for their utility through the direct transfer processes. It is, however, crucial to also consider the hybridization of the surface electronic states with acceptor states, where the latter can originate, for example, from an adsorbed molecule ${ }^{67}$ or a semiconductor. ${ }^{68,69}$ To maximize the efficiency for direct excitation transfer, the emitting and receiving states should be energetically aligned and spatially overlapping. In addition, hotelectron generation can be affected by the dielectric environment, for example, by red-shifting the plasmon resonance so that $\mathrm{d}$-band electrons are not excited. The framework presented here enables analysis and quantification of these aspects at the atomic scale with material specificity and without resorting to empirical parameters.

\section{CONCLUSIONS}

In conclusion, we presented a comprehensive first-principles account of the real-time dynamics of plasmon formation and dephasing into HCs and analyzed quantitatively the impact of atomic-scale structure on the $\mathrm{HC}$ generation. Our results on silver NPs indicate that lower-coordinated surface sites exhibit a larger proportion of hot electrons, especially those with higher energy, than the bulk of the nanoparticle or higher-coordinated surface sites. In contrast, the distribution of hot holes is relatively homogeneous within each considered NP. These features can be traced to the electronic structure as hole and electron states 
exhibit localized $\mathrm{d}$ - and delocalized sp-type character, respectively. We would therefore expect the present insight to be qualitatively transferable to other late transition metals that exhibit similar electronic structure. The observed trends are present in NPs of different shapes and sizes with varying prevalence. We therefore envision that the obtained atomicscale insights could be applicable to nanoscale structures in general. First-principles predictions of plasmonic HC generation, as presented here, can thereby facilitate tuning and optimizing photocatalytic systems down to the atomic scale, for example, by enabling identification of promising surfaceacceptor combinations.

\section{METHODS}

Computational Details. The ground-state electronic structures were calculated with KS-DFT ${ }^{36,37}$ using the GLLB-sc exchangecorrelation potential. ${ }^{40,41}$ The time-domain responses were calculated with TDDFT $^{38}$ starting from the ground state. The dynamical response was described with RPA for the data in Figures 1 and 2 and in Supplementary Figures S1-S6, whereas all the other data were calculated with the adiabatic GLLB-sc. ${ }^{28}$ The two response kernels yield very similar results (Supplementary Figure S10), but the GLLB-sc potential is not suitable for obtaining the total energies.

All of the calculations were carried out with the open-source GPAW code package ${ }^{70}$ using localized basis sets ${ }^{71}$ and the real-time propagation TDDFT implementation. ${ }^{28}$ We used 11-electron projector-augmented wave ${ }^{72}$ setups for $\mathrm{Ag}$, treating the remaining electrons as a frozen core. We used an extended "p-valence" basis set that includes diffuse $5 p$ functions, which are important for describing plasmon resonances. ${ }^{73}$ The basis set is similar to the ones used in refs 28 and 39.

The photoabsorption spectra were calculated using the $\delta$-kick technique, ${ }^{74}$ yielding linear impulse response. The photoabsorption of icosahedral particles is isotropic, and the electric field was aligned along the $x$ direction. The resulting HCs do not exhibit a strong variation between different sites (Figure 3c). For the time propagation, we used a time step of 10 as and total propagation time of at least $30 \mathrm{fs}$. The spectra were broadened using Gaussian damping with $\sigma=0.07 \mathrm{eV}$ corresponding to a full width at half-maximum of $0.16 \mathrm{eV}$. The real-time response to a pulse was calculated as a postprocessing step through convolution as described below in detail. In the convolution Fourier transforms or time-domain response, there is no artificial damping.

A grid spacing parameter of $h=0.3 \AA$ was chosen to represent densities and potentials, and the nanoparticles were surrounded by a vacuum region of at least $6 \AA$. The Hartree potential was evaluated with a Poisson solver using the monopole and dipole corrections for the potential. Fermi-Dirac smearing was applied to the occupation numbers to facilitate convergence. The KS electron-hole basis included electron-hole pairs with occupation number difference $f_{i}-$ $f_{a} \geq 10^{-3}$.

Before the response calculations, all geometries were relaxed using the BFGS optimizer in the open-source ASE package. ${ }^{75}$ The relaxation calculations used the Perdew-Burke-Ernzerhof $(\mathrm{PBE})^{76}$ functional, double- $\zeta$ polarized (dzp) basis sets, and $h=0.2 \AA$.

Pulse Response from Convolution. The photoabsorption spectrum can be efficiently calculated from real-time propagation using the $\delta$-kick perturbation ${ }^{74}$ as in the linear-response regime all the frequencies are independent of each other. We utilize this property in the present work for calculating the linear response of the density matrix to the Gaussian pulse of eq 1 as a postprocessing step. First, the time propagation is carried out for perturbation $v_{\text {kick }}(t)=z K_{0} \delta(t)$, yielding the impulse response of the system and the corresponding timedependent density matrix $\delta \rho_{i a}^{\text {kick }}(t)$. Then, in the linear-response regime, the response to the pulse of eq 1 is obtained as a convolution

$$
\delta \rho_{i a}(t)=\frac{1}{K_{0}} \int_{0}^{\infty} \delta \rho_{i a}^{\text {kick }}(\tau) \mathcal{E}(t-\tau) \mathrm{d} \tau
$$

which can be very efficiently calculated in frequency space by employing the convolution theorem and inverse Fourier transform

$$
\delta \rho_{i a}(t)=\frac{1}{2 \pi K_{0}} \int_{-\infty}^{\infty} \delta \rho_{i a}^{\text {kick }}(\omega) \mathcal{E}(\omega) \mathrm{e}^{-i \omega t} \mathrm{~d} \omega
$$

where $\delta \rho_{i a}^{\text {kick }}(\omega)$ and $\mathcal{E}(\omega)$ are Fourier transforms of the respective time-domain quantities. Here, $\delta \rho_{i a}^{\text {kick }}(\omega)$ can be efficiently calculated from the impulse response by using the computational framework developed in ref 39. The time derivatives required for calculating the energy (Supplementary Note S4) are obtained similarly as

$$
\begin{aligned}
& \delta \dot{\rho}_{i a}(t)=-\frac{i}{2 \pi K_{0}} \int_{-\infty}^{\infty} \omega \delta \rho_{i a}^{\text {kick }}(\omega) \mathcal{E}(\omega) \mathrm{e}^{-i \omega t} \mathrm{~d} \omega \\
& \delta \ddot{\rho}_{i a}(t)=-\frac{1}{2 \pi K_{0}} \int_{-\infty}^{\infty} \omega^{2} \delta \rho_{i a}^{\text {kick }}(\omega) \mathcal{E}(\omega) \mathrm{e}^{-i \omega t} \mathrm{~d} \omega
\end{aligned}
$$

In practice, $\mathcal{E}(\omega)$ is nonvanishing only on a finite frequency interval (see Figure 1a), which narrows the integration limits.

It is emphasized that the time-dependent density matrix, $\delta \rho_{i a}(t)$, is a complex quantity in time domain, so in practical calculations, it is convenient to carry out Fourier transform for the real $\operatorname{Re} \delta \rho_{i a}(t)$ and imaginary $\operatorname{Im} \delta \rho_{i a}(t)$ parts separately to utilize the properties of Fourier transform of real quantities.

We also note in passing that the impulse response $\delta \rho_{i a}^{\text {kick }}(\omega)$ can be equivalently calculated from the Casida linear-response frequencyspace formalism. ${ }^{39,77}$ Hence, the linear real-time response to any pulse can also be calculated from the Casida solutions by using the convolution of eq 8.

Total Absorbed Energy. By invoking Fourier transform, the total absorbed energy after the pulse has vanished is obtained as

$$
\int_{0}^{\infty} \delta \dot{\mu}(t) \mathcal{E}(t) \mathrm{d} t=\frac{1}{2} \int_{0}^{\infty} S(\omega)|\mathcal{E}(\omega)|^{2} \mathrm{~d} \omega
$$

where $S(\omega)=\frac{2 \omega}{\pi} \operatorname{Im}[\alpha(\omega)]$ is the dipole strength function, which equals the photoabsorption cross section safe for a constant multiplier.

Hot-Carrier Distributions. The hot-electron energy distributions corresponding to the state occupation probabilities $P_{a}^{\mathrm{e}}$ of eq 6 are obtained as (time dependence is not explicitly marked)

$$
P_{\mathrm{e}}(\epsilon)=\sum_{a} P_{a}^{\mathrm{e}} \delta\left(\epsilon-\epsilon_{a}\right)=\frac{1}{2} \sum_{i a}^{f_{i}>f_{a}}\left(q_{i a}^{2}+p_{i a}^{2}\right) \delta\left(\epsilon-\epsilon_{a}\right)
$$

where

$$
q_{i a}(t)=2 \operatorname{Re} \delta \rho_{i a}(t) / \sqrt{2\left(f_{i}-f_{a}\right)}
$$

and

$$
p_{i a}(t)=-2 \operatorname{Im} \delta \rho_{i a}(t) / \sqrt{2\left(f_{i}-f_{a}\right)}
$$

that is, they correspond to the real and imaginary parts of $\delta \rho_{i a}$ (see Supplementary Note S2 for details). For visualization purposes, Gaussian smoothing (convolution) is applied with respect to the $\epsilon$ axis.

The spatial probability density of hot electrons is given by the full electron-electron part of the second-order density matrix as (see Supplementary Note $S 1$ and note that only the real part contributes due to the hermiticity of the density matrix)

$$
P_{\mathrm{e}}(\boldsymbol{r})=\frac{1}{2} \sum_{i a a^{\prime}}^{\substack{f_{i}>f_{a} \\ f_{a}=f_{a \prime}}}\left(q_{i a} q_{i a,}+p_{i a} p_{i a}\right) \psi_{a}^{(0)}(\boldsymbol{r}) \psi_{a \prime}^{(0)}(\boldsymbol{r})
$$

The diagonal and degenerate states dominate the spatial density contributions, which allows us to define a spatio-energetic distribution

$$
P_{\mathrm{e}}(\epsilon, \boldsymbol{r})=\frac{1}{2} \sum_{i a a^{\prime}}^{\substack{f_{i}>f_{a} \\ \epsilon_{a}=\epsilon_{a \prime}}}\left(q_{i a} q_{i a \prime}+p_{i a} p_{i a \prime}\right) \psi_{a}^{(0)}(\boldsymbol{r}) \psi_{a \prime}^{(0)}(\boldsymbol{r}) \delta\left(\epsilon-\epsilon_{a}\right)
$$


which is used to calculate the spatial density of hot electrons with more than $1 \mathrm{eV}$ as $P_{\mathrm{e}}^{>\mathrm{leV}}(\boldsymbol{r})=\int_{\mathrm{leV}}^{\infty} P_{\mathrm{e}}(\epsilon, \boldsymbol{r}) \mathrm{d} \epsilon$, and the energy distribution of hot electrons in a spatial volume $V$ as $P_{\mathrm{e}}^{V}(\epsilon)=\int_{V} P_{\mathrm{e}}(\epsilon, \boldsymbol{r}) \mathrm{d} \boldsymbol{r}$. The distribution at a specific atomic site (e.g., corner atoms) is obtained by integration over the Voronoi cell associated with the site.

The spatial and energetic distributions of hot holes are calculated analogously to the electrons.

Software Used. The GPAW package ${ }^{70,78}$ with linear combination of atomic orbitals (LCAO) mode ${ }^{1}$ was used for DFT calculations. The real-time propagation LCAO-TDDFT implementation in $\mathrm{GPAW}^{28}$ was used for the TDDFT calculations. Density-matrix-based analysis tools in frequency space ${ }^{39}$ and in real time (present work) were used for analysis. The ASE library ${ }^{75}$ was used for constructing atomic structures and geometry relaxation. The NumPy ${ }^{79}$ and Matplotlib ${ }^{80}$ Python packages and the VMD software ${ }^{81,82}$ were used for processing and plotting data.

\section{ASSOCIATED CONTENT}

\section{(s) Supporting Information}

The Supporting Information is available free of charge at https://pubs.acs.org/doi/10.1021/acsnano.0c03004.

Figures S1-S10 displaying supporting data, notes S1-S4 deriving equations, and supporting reference (PDF)

\section{AUTHOR INFORMATION}

\section{Corresponding Authors}

Tuomas P. Rossi - Department of Physics, Chalmers University

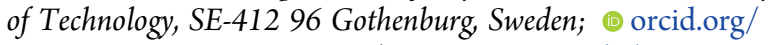
0000-0002-8713-4559; Email: tuomas.rossi@chalmers.se

Paul Erhart - Department of Physics, Chalmers University of Technology, SE-412 96 Gothenburg, Sweden; 자이.org/ 0000-0002-2516-6061; Email: erhart@chalmers.se

Mikael Kuisma - Department of Chemistry, Nanoscience Center, University of Jyväskylä, FI-40014 Jyväskylä, Finland; 다이.org/0000-0001-8323-3405; Email: mikael.j.kuisma@ jyu.fi

Complete contact information is available at: https://pubs.acs.org/10.1021/acsnano.0c03004

\section{Notes}

The authors declare no competing financial interest. Scripts, input and data files are also available on Zenodo (https://doi.org/10.5281/zenodo.3927527).

\section{ACKNOWLEDGMENTS}

We acknowledge financial support from the Knut and Alice Wallenberg Foundation (2014.0226, 2015.0055), the Swedish Research Council (2015-04153), and the Swedish Foundation for Strategic Research (RMA15-0052). T.P.R. acknowledges support from the European Union's Horizon 2020 research and innovation programme under the Marie Skłodowska-Curie Grant Agreement No. 838996 and also thanks the Adlerbert Research Foundation and the Wilhelm and Martina Lundgren Foundation for support. M.K. acknowledges funding from Academy of Finland under Grant No. 295602. We acknowledge generous computational resources provided by the Swedish National Infrastructure for Computing (SNIC) at PDC (Stockholm), NSC (Linköping), and C3SE (Gothenburg) as well as by the CSC-IT Center for Science (Finland).

\section{REFERENCES}

(1) Aslam, U.; Rao, V. G.; Chavez, S.; Linic, S. Catalytic Conversion of Solar to Chemical Energy on Plasmonic Metal Nanostructures. Nat. Catal. 2018, 1, 656.

(2) Linic, S.; Aslam, U.; Boerigter, C.; Morabito, M. Photochemical Transformations on Plasmonic Metal Nanoparticles. Nat. Mater. 2015, 14, 567.

(3) Brongersma, M. L.; Halas, N. J.; Nordlander, P. Plasmon-Induced Hot Carrier Science and Technology. Nat. Nanotechnol. 2015, 10, 25.

(4) Saavedra, J. R. M.; Asenjo-Garcia, A.; García de Abajo, F. J. HotElectron Dynamics and Thermalization in Small Metallic Nanoparticles. ACS Photonics 2016, 3, 1637.

(5) Liu, J. G.; Zhang, H.; Link, S.; Nordlander, P. Relaxation of Plasmon-Induced Hot Carriers. ACS Photonics 2018, 5, 2584.

(6) Atwater, H. A.; Polman, A. Plasmonics for Improved Photovoltaic Devices. Nat. Mater. 2010, 9, 205.

(7) Knight, M. W.; Sobhani, H.; Nordlander, P.; Halas, N. J. Photodetection with Active Optical Antennas. Science 2011, 332, 702.

(8) Chalabi, H.; Schoen, D.; Brongersma, M. L. Hot-Electron Photodetection with a Plasmonic Nanostripe Antenna. Nano Lett. 2014, 14, 1374.

(9) Naik, G. V.; Welch, A. J.; Briggs, J. A.; Solomon, M. L.; Dionne, J. A. Hot-Carrier-Mediated Photon Upconversion in Metal-Decorated Quantum Wells. Nano Lett. 2017, 17, 4583.

(10) Mukherjee, S.; Libisch, F.; Large, N.; Neumann, O.; Brown, L. V.; Cheng, J.; Lassiter, J. B.; Carter, E. A.; Nordlander, P.; Halas, N. J. Hot Electrons Do the Impossible: Plasmon-Induced Dissociation of $\mathrm{H}_{2}$ on Au. Nano Lett. 2013, 13, 240.

(11) Mubeen, S.; Lee, J.; Singh, N.; Krämer, S.; Stucky, G. D.; Moskovits, M. An Autonomous Photosynthetic Device in which All Charge Carriers Derive from Surface Plasmons. Nat. Nanotechnol. 2013, 8, 247.

(12) Kale, M. J.; Avanesian, T.; Christopher, P. Direct Photocatalysis by Plasmonic Nanostructures. ACS Catal. 2014, 4, 116.

(13) Swearer, D. F.; Zhao, H.; Zhou, L.; Zhang, C.; Robatjazi, H.; Martirez, J. M. P.; Krauter, C. M.; Yazdi, S.; McClain, M. J.; Ringe, E.; Carter, E. A.; Nordlander, P.; Halas, N. J. Heterometallic AntennaReactor Complexes for Photocatalysis. Proc. Natl. Acad. Sci. U. S. A. 2016, 113, 8916.

(14) Zhai, Y.; DuChene, J. S.; Wang, Y.-C.; Qiu, J.; Johnston-Peck, A. C.; You, B.; Guo, W.; DiCiaccio, B.; Qian, K.; Zhao, E. W.; Ooi, F.; Hu, D.; Su, D.; Stach, E. A.; Zhu, Z.; Wei, W. D. PolyvinylpyrrolidoneInduced Anisotropic Growth of Gold Nanoprisms in Plasmon-Driven Synthesis. Nat. Mater. 2016, 15, 889.

(15) Zhou, L.; Swearer, D. F.; Zhang, C.; Robatjazi, H.; Zhao, H.; Henderson, L.; Dong, L.; Christopher, P.; Carter, E. A.; Nordlander, P.; Halas, N. J. Quantifying Hot Carrier and Thermal Contributions in Plasmonic Photocatalysis. Science 2018, 362, 69.

(16) Sivan, Y.; Baraban, J.; Un, I. W.; Dubi, Y. Comment on "Quantifying Hot Carrier and Thermal Contributions in Plasmonic Photocatalysis. Science 2019, 364, No. eaaw9367.

(17) Dubi, Y.; Sivan, Y. Hot" Electrons in Metallic NanostructuresNon-Thermal Carriers or Heating? Light: Sci. Appl. 2019, 8, 89.

(18) Sundararaman, R.; Narang, P.; Jermyn, A. S.; Goddard, W. A.; Atwater, H. A. Theoretical Predictions for Hot-Carrier Generation from Surface Plasmon Decay. Nat. Commun. 2014, 5, 5788.

(19) Bernardi, M.; Mustafa, J.; Neaton, J. B.; Louie, S. G. Theory and Computation of Hot Carriers Generated by Surface Plasmon Polaritons in Noble Metals. Nat. Commun. 2015, 6, 7044.

(20) Manjavacas, A.; Liu, J. G.; Kulkarni, V.; Nordlander, P. PlasmonInduced Hot Carriers in Metallic Nanoparticles. ACS Nano 2014, 8, 7630.

(21) Besteiro, L. V.; Govorov, A. O. Amplified Generation of Hot Electrons and Quantum Surface Effects in Nanoparticle Dimers with Plasmonic Hot Spots. J. Phys. Chem. C 2016, 120, 19329.

(22) Yan, L.; Wang, F.; Meng, S. Quantum Mode Selectivity of Plasmon-Induced Water Splitting on Gold Nanoparticles. ACS Nano 2016, 10, 5452 . 
(23) Dal Forno, S.; Ranno, L.; Lischner, J. Material, Size, and Environment Dependence of Plasmon-Induced Hot Carriers in Metallic Nanoparticles. J. Phys. Chem. C 2018, 122, 8517.

(24) Ranno, L.; Dal Forno, S.; Lischner, J. Computational Design of Bimetallic Core-Shell Nanoparticles for Hot-Carrier Photocatalysis. npj. Comput. Mater. 2018, 4, 31.

(25) Román Castellanos, L.; Hess, O.; Lischner, J. Single Plasmon Hot Carrier Generation in Metallic Nanoparticles. Commun. Phys. 2019, 2, 47.

(26) Román Castellanos, L.; Kahk, J. M.; Hess, O.; Lischner, J. Generation of Plasmonic Hot Carriers from D-Bands in Metallic Nanoparticles. J. Chem. Phys. 2020, 152, 104111.

(27) Zhang, P.; Feist, J.; Rubio, A.; García-González, P.; García-Vidal, F. J. Ab Initio Nanoplasmonics: The Impact of Atomic Structure. Phys. Rev. B: Condens. Matter Mater. Phys. 2014, 90, 161407.

(28) Kuisma, M.; Sakko, A.; Rossi, T. P.; Larsen, A. H.; Enkovaara, J.; Lehtovaara, L.; Rantala, T. T. Localized Surface Plasmon Resonance in Silver Nanoparticles: Atomistic First-Principles Time-Dependent Density-Functional Theory Calculations. Phys. Rev. B: Condens. Matter Mater. Phys. 2015, 91, 115431.

(29) Rossi, T. P.; Zugarramurdi, A.; Puska, M. J.; Nieminen, R. M. Quantized Evolution of the Plasmonic Response in a Stretched Nanorod. Phys. Rev. Lett. 2015, 115, 236804.

(30) Marchesin, F.; Koval, P.; Barbry, M.; Aizpurua, J.; SánchezPortal, D. Plasmonic Response of Metallic Nanojunctions Driven by Single Atom Motion: Quantum Transport Revealed in Optics. ACS Photonics 2016, 3, 269.

(31) Donati, G.; Lingerfelt, D. B.; Aikens, C. M.; Li, X. Molecular Vibration Induced Plasmon Decay. J. Phys. Chem. C 2017, 121, 15368. (32) Senanayake, R. D.; Lingerfelt, D. B.; Kuda-Singappulige, G. U.; Li, X.; Aikens, C. M. Real-Time TDDFT Investigation of Optical Absorption in Gold Nanowires. J. Phys. Chem. C 2019, 123, 14734.

(33) Ma, J.; Wang, Z.; Wang, L.-W. Interplay between Plasmon and Single-Particle Excitations in a Metal Nanocluster. Nat. Commun. 2015, 6, 10107.

(34) Douglas-Gallardo, O. A.; Berdakin, M.; Sánchez, C. G. Atomistic Insights into Chemical Interface Damping of Surface Plasmon Excitations in Silver Nanoclusters. J. Phys. Chem. C 2016, 120, 24389.

(35) Douglas-Gallardo, O. A.; Berdakin, M.; Frauenheim, T.; Sánchez,

C. G. Plasmon-Induced Hot-Carrier Generation Differences in Gold and Silver Nanoclusters. Nanoscale 2019, 11, 8604.

(36) Hohenberg, P.; Kohn, W. Inhomogeneous Electron Gas. Phys. Rev. 1964, 136, B864.

(37) Kohn, W.; Sham, L. J. Self-Consistent Equations including Exchange and Correlation Effects. Phys. Rev. 1965, 140, A1 133.

(38) Runge, E.; Gross, E. K. U. Density-Functional Theory for TimeDependent Systems. Phys. Rev. Lett. 1984, 52, 997.

(39) Rossi, T. P.; Kuisma, M.; Puska, M. J.; Nieminen, R. M.; Erhart, P. Kohn-Sham Decomposition in Real-Time Time-Dependent DensityFunctional Theory: An Efficient Tool for Analyzing Plasmonic Excitations. J. Chem. Theory Comput. 2017, 13, 4779.

(40) Gritsenko, O.; van Leeuwen, R.; van Lenthe, E.; Baerends, E. J. Self-Consistent Approximation to the Kohn-Sham Exchange Potential. Phys. Rev. A: At., Mol., Opt. Phys. 1995, 51, 1944.

(41) Kuisma, M.; Ojanen, J.; Enkovaara, J.; Rantala, T. Kohn-Sham Potential with Discontinuity for Band Gap Materials. Phys. Rev. B: Condens. Matter Mater. Phys. 2010, 82, 115106.

(42) Yan, J.; Jacobsen, K. W.; Thygesen, K. S. First-Principles Study of Surface Plasmons on $\mathrm{Ag}(111)$ and $\mathrm{H} / \mathrm{Ag}(111)$. Phys. Rev. B: Condens. Matter Mater. Phys. 2011, 84, 235430.

(43) Yannouleas, C.; Broglia, R. Landau Damping and Wall Dissipation in Large Metal Clusters. Ann. Phys. 1992, 217, 105.

(44) Bernadotte, S.; Evers, F.; Jacob, C. R. Plasmons in Molecules. J. Phys. Chem. C 2013, 117, 1863.

(45) Yannouleas, C.; Broglia, R. A.; Brack, M.; Bortignon, P. F. Fragmentation of the Photoabsorption Strength in Neutral and Charged Metal Microclusters. Phys. Rev. Lett. 1989, 63, 255.
(46) Yannouleas, C.; Broglia, R. A. Collective and Single-Particle Aspects in the Optical Response of Metal Microclusters. Phys. Rev. A: At., Mol., Opt. Phys. 1991, 44, 5793.

(47) You, X.; Ramakrishna, S.; Seideman, T. Origin of Plasmon Lineshape and Enhanced Hot Electron Generation in Metal Nanoparticles. J. Phys. Chem. Lett. 2018, 9, 141.

(48) Zhang, R.; Bursi, L.; Cox, J. D.; Cui, Y.; Krauter, C. M.; Alabastri, A.; Manjavacas, A.; Calzolari, A.; Corni, S.; Molinari, E.; Carter, E. A.; García de Abajo, F. J.; Zhang, H.; Nordlander, P. How to Identify Plasmons from the Optical Response of Nanostructures. ACS Nano 2017, 11, 7321.

(49) Hartland, G. V.; Besteiro, L. V.; Johns, P.; Govorov, A. O. What's So Hot about Electrons in Metal Nanoparticles? ACS Energy Lett. 2017, 2,1641 .

(50) Aizpurua, J.; Baletto, F.; Baumberg, J.; Christopher, P.; Nijs, B. d.; Deshpande, P.; Diaz Fernandez, Y.; Fabris, L.; Freakley, S.; Gawinkowski, S.; Govorov, A.; Halas, N.; Hernandez, R.; Jankiewicz, B.; Khurgin, J.; Kuisma, M.; Kumar, P. V.; Lischner, J.; Liu, J.; Marini, A.; et al. Theory of Hot Electrons: General Discussion. Faraday Discuss. 2019, 214, 245 .

(51) Khurgin, J. B. Fundamental Limits of Hot Carrier Injection from Metal in Nanoplasmonics. Nanophotonics 2020, 9, 453.

(52) Brown, A. M.; Sundararaman, R.; Narang, P.; Goddard, W. A.; Atwater, H. A. Nonradiative Plasmon Decay and Hot Carrier Dynamics: Effects of Phonons, Surfaces, and Geometry. ACS Nano 2016, 10, 957.

(53) Narang, P.; Sundararaman, R.; Atwater, H. A. Plasmonic Hot Carrier Dynamics in Solid-State and Chemical Systems for Energy Conversion. Nanophotonics 2016, 5, 96.

(54) Christopher, P.; Moskovits, M. Hot Charge Carrier Transmission from Plasmonic Nanostructures. Annu. Rev. Phys. Chem. 2017, 68, 379.

(55) Wu, K.; Chen, J.; McBride, J. R.; Lian, T. Efficient Hot-Electron Transfer by a Plasmon-Induced Interfacial Charge-Transfer Transition. Science 2015, 349, 632.

(56) Kale, M. J.; Christopher, P. Plasmons at the Interface. Science 2015, 349, 587.

(57) Boerigter, C.; Campana, R.; Morabito, M.; Linic, S. Evidence and Implications of Direct Charge Excitation as the Dominant Mechanism in Plasmon-Mediated Photocatalysis. Nat. Commun. 2016, 7, 10545.

(58) Boerigter, C.; Aslam, U.; Linic, S. Mechanism of Charge Transfer from Plasmonic Nanostructures to Chemically Attached Materials. ACS Nano 2016, 10, 6108.

(59) Tan, S.; Argondizzo, A.; Ren, J.; Liu, L.; Zhao, J.; Petek, H. Plasmonic Coupling at a Metal/Semiconductor Interface. Nat. Photonics 2017, 11, 806.

(60) Tan, S.; Dai, Y.; Zhang, S.; Liu, L.; Zhao, J.; Petek, H. Coherent Electron Transfer at the $\mathrm{Ag} / \mathrm{Graphite}$ Heterojunction Interface. Phys. Rev. Lett. 2018, 120, 126801.

(61) Li, Y.; DiStefano, J. G.; Murthy, A. A.; Cain, J. D.; Hanson, E. D.; Li, Q.; Castro, F. C.; Chen, X.; Dravid, V. P. Superior Plasmonic Photodetectors Based on $\mathrm{Au} @ \mathrm{MoS}_{2}$ Core-Shell Heterostructures. ACS Nano 2017, 11, 10321.

(62) Foerster, B.; Joplin, A.; Kaefer, K.; Celiksoy, S.; Link, S.; Sönnichsen, C. Chemical Interface Damping Depends on Electrons Reaching the Surface. ACS Nano 2017, 11, 2886.

(63) Ratchford, D. C.; Dunkelberger, A. D.; Vurgaftman, I.; Owrutsky, J. C.; Pehrsson, P. E. Quantification of Efficient Plasmonic HotElectron Injection in Gold Nanoparticle- $\mathrm{TiO}_{2}$ Films. Nano Lett. 2017, $17,6047$.

(64) Tan, S.; Liu, L.; Dai, Y.; Ren, J.; Zhao, J.; Petek, H. Ultrafast Plasmon-Enhanced Hot Electron Generation at Ag Nanocluster/ Graphite Heterojunctions. J. Am. Chem. Soc. 2017, 139, 6160.

(65) Brown, A. M.; Sundararaman, R.; Narang, P.; Schwartzberg, A. M.; Goddard, W. A.; Atwater, H. A. Experimental and Ab Initio Ultrafast Carrier Dynamics in Plasmonic Nanoparticles. Phys. Rev. Lett. 2017, 118 , No. 087401.

(66) Besteiro, L. V.; Kong, X.-T.; Wang, Z.; Hartland, G.; Govorov, A. O. Understanding Hot-Electron Generation and Plasmon Relaxation in 
Metal Nanocrystals: Quantum and Classical Mechanisms. ACS Photonics 2017, 4, 2759.

(67) Kumar, P. V.; Rossi, T. P.; Kuisma, M.; Erhart, P.; Norris, D. J. Direct Hot-Carrier Transfer in Plasmonic Catalysis. Faraday Discuss. 2019, 214, 189.

(68) Kumar, P. V.; Rossi, T. P.; Marti-Dafcik, D.; Reichmuth, D.; Kuisma, M.; Erhart, P.; Puska, M. J.; Norris, D. J. Plasmon-Induced Direct Hot-Carrier Transfer at Metal-Acceptor Interfaces. ACS Nano 2019, 13, 3188.

(69) Ma, J.; Gao, S. Plasmon-Induced Electron-Hole Separation at the $\mathrm{Ag} / \mathrm{TiO}_{2}(110)$ Interface. ACS Nano 2019, 13, 13658.

(70) Enkovaara, J.; Rostgaard, C.; Mortensen, J. J.; Chen, J.; Dulak, M.; Ferrighi, L.; Gavnholt, J.; Glinsvad, C.; Haikola, V.; Hansen, H. A.; Kristoffersen, H. H.; Kuisma, M.; Larsen, A. H.; Lehtovaara, L.; Ljungberg, M.; Lopez-Acevedo, O.; Moses, P. G.; Ojanen, J.; Olsen, T.; Petzold, V.; et al. Electronic Structure Calculations with GPAW: A Real-Space Implementation of the Projector Augmented-Wave Method. J. Phys.: Condens. Matter 2010, 22, 253202.

(71) Larsen, A. H.; Vanin, M.; Mortensen, J. J.; Thygesen, K. S.; Jacobsen, K. W. Localized Atomic Basis Set in the Projector Augmented Wave Method. Phys. Rev. B: Condens. Matter Mater. Phys. 2009, 80, 195112.

(72) Blöchl, P. E. Projector Augmented-Wave Method. Phys. Rev. B: Condens. Matter Mater. Phys. 1994, 50, 17953.

(73) Rossi, T. P.; Lehtola, S.; Sakko, A.; Puska, M. J.; Nieminen, R. M. Nanoplasmonics Simulations at the Basis Set Limit through Completeness-Optimized, Local Numerical Basis Sets. J. Chem. Phys. 2015, 142, No. 094114.

(74) Yabana, K.; Bertsch, G. F. Time-Dependent Local-Density Approximation in Real Time. Phys. Rev. B: Condens. Matter Mater. Phys. 1996, 54, 4484.

(75) Larsen, A.; Mortensen, J.; Blomqvist, J.; Castelli, I.; Christensen, R.; Dulak, M.; Friis, J.; Groves, M.; Hammer, B.; Hargus, C.; Hermes, E.; Jennings, P.; Jensen, P.; Kermode, J.; Kitchin, J.; Kolsbjerg, E.; Kubal, J.; Kaasbjerg, K.; Lysgaard, S.; Maronsson, J.; et al. The Atomic Simulation Environment - A Python Library for Working with Atoms. J. Phys.: Condens. Matter 2017, 29, 273002.

(76) Perdew, J. P.; Burke, K.; Ernzerhof, M. Generalized Gradient Approximation Made Simple. Phys. Rev. Lett. 1996, 77, 3865.

(77) Casida, M. E. Time-Dependent Density Functional Response Theory for Molecules. In Recent Advances in Density Functional Methods, Part I; Chong, D. P., Ed.; World Scientific: Singapore, 1995; p 155.

(78) Mortensen, J. J.; Hansen, L. B.; Jacobsen, K. W. Real-Space Grid Implementation of the Projector Augmented Wave Method. Phys. Rev. B: Condens. Matter Mater. Phys. 2005, 71, No. 035109.

(79) van der Walt, S.; Colbert, S. C.; Varoquaux, G. The NumPy Array: A Structure for Efficient Numerical Computation. Comput. Sci. Eng. 2011, 13, 22.

(80) Hunter, J. D. Matplotlib: A 2D Graphics Environment. Comput. Sci. Eng. 2007, 9, 90.

(81) Humphrey, W.; Dalke, A.; Schulten, K. VMD - Visual Molecular Dynamics. J. Mol. Graphics 1996, 14, 33.

(82) Stone, J. An Efficient Library for Parallel Ray Tracing and Animation. M.Sc. Thesis, University of Missouri-Rolla, 1998. 